\title{
Application of Restarted Adomian Decomposition Method on Nonlinear System of Integro-Differential Equations
}

\author{
H. O. Bakodah ${ }^{1}$, M. Al-Mazmumy ${ }^{1}$, S. O. Almuhalbedi ${ }^{2}$ \\ ${ }^{1}$ Department of mathematics, Faculty of Science-AL Faisaliah Campus, King Abdulaziz University, Jeddah, \\ Saudi Arabia. \\ 2 Department of Mathematics; Faculty of Science; King Abdulaziz University, Jeddah; Saudi Arabia. \\ * Corresponding author. Email: hbakodah@kau.edu.sa \\ Manuscript submitted June 12, 2017; accepted September 2, 2017. \\ doi: 10.17706/ijapm.2017.7.4.216-223
}

\begin{abstract}
In this paper, the Restarted Adomian Method has been implemented to analyze the System of Integro-Differential Equations in both Volterra and Fredholm types. This modification of Adomian decomposition method is much better than the standard version of the Adomian decomposition method, where more accurate approximations for the solutions of the studied examples have been achieved.
\end{abstract}

Key words: System of volterra integro-differential equation, system of fredholm integro-differential equation, adomian decomposition method, restarted adomian method.

\section{Introduction}

The 'Adomian Decomposition Method' (ADM) [1] consists of calculating the function $u$ as a series $u=\sum_{n=0}^{\infty} u_{n}$, and the nonlinear function is decomposed as $N(u)=N\left(\sum_{n=0}^{\infty} A_{n} x^{n}\right)$, where the $A_{n}$, are given by $A_{n}=\left.\sum_{n=0}^{\infty} c(v, n) N^{(v)}(u)\right|_{u=u_{0}}$ [2]. In computing the series $u$ using appropriate software, as $\mathrm{n}$ increases the number of terms in the expression for $A_{n}$ increases and this causes propagation of round off errors. On the other hand, the factor $\frac{1}{n !}$, existing in the formula of $A_{n}$, makes it very small, so that its contribution to $u$ is negligible. Hence, only the first few terms of the series $\sum_{n=0}^{\infty} u_{n}$ determine the accuracy of the approximate solution. In 2003, E. Babolian, et al. [3] paid attention to this fact and introduced a new algorithm called 'Restarted Adomian Method', based on the 'Adomian Decomposition Method' to improve the accuracy dramatically. The 'Restarted Adomian Decomposition Method' (RADM), based on the standard ADM, introduced by E.Babolian, et al. [3] was for algebraic equations. In 2008, A. Yahidi et al. [4] described the 'Restarted Adomian Decomposition Method' to solve the system of nonlinear algebraic equations. In 2005, E. Babolian, et al. [5] applied the 'Restarted Adomian Decomposition Method' for solving nonlinear differential equation. In 2004, E. Babolian, et al. [6] applied the (RADM) to nonlinear integral equations and integro-differential equation. H. Sadeghi, et al. [7] applied the RADM for solving system of nonlinear Volterra integral equations. In this paper we will show by examples that convergence rate this method is more accelerate than standard ADM for solving System of Integro-Differential Equations in both Volterra and Fredholm types.

\section{Restarted Adomian Decomposition Method}

We consider the Integro-differentil equation of the form 


$$
\left\{\begin{array}{l}
u^{\prime \prime}(x)=f_{1}(x)+\int_{a}^{b(x)}\left(k_{1}(x, t) N_{1}(u(t))+k_{1}^{\sim}(x, t) N_{1}^{\sim}(v(t))\right) d t \\
v^{\prime \prime}(x)=f_{2}(x)+\int_{a}^{b(x)}\left(k_{2}(x, t) N_{2}(u(t))+k_{2}^{\sim}(x, t) N_{2}^{\sim}(v(t))\right) d t
\end{array}\right.
$$

with initial condotions:

$$
\begin{array}{ll}
u(0)=\alpha_{1}, & u^{\prime}(0)=\beta_{1} . \\
v(0)=\alpha_{2}, & v^{\prime}(0)=\beta_{2}
\end{array}
$$

where $\mathrm{u}^{\prime}{ }^{\prime}(\mathrm{x}), v^{\prime \prime}(x)$ are the second derivative of the unknown function $\mathrm{u}(\mathrm{x}), v(x)$ that will be determined, $k_{1}(x, t), k_{1}^{\sim}(x, t), k_{2}(x, t)$ and $k_{2}^{\sim}(x, t)$ are the kernels of the integro differential equations, $f_{1}(x), f_{2}(x)$ are an analytic function, $N_{1}(u(t)), N_{2}(u(t)), N_{1}^{\sim}(v(t)), N_{2}^{\sim}(v(t))$ are nonlinear function of u, $v$ respectively.

Let $L=\frac{d^{2}}{d x^{2}}$, so $L^{-1}()=.\int_{0}^{x} \int_{0}^{x}() d x d$.$x , applying L^{-1}$ to both sides of (1), and using initial conditions, we obtain

$$
\left\{\begin{array}{l}
u(x)=\alpha_{1}+\beta_{1} x+L^{-1} f_{1}(x)+L^{-1} \int_{a}^{b(x)}\left(k_{1}(x, t) N_{1}(u(t))+k_{1}^{\sim}(x, t) N_{1}^{\sim}(v(t))\right) d t \\
v(x)=\alpha_{2}+\beta_{2} x+L^{-1} f_{2}(x)+L^{-1} \int_{a}^{b(x)}\left(k_{2}(x, t) N_{2}(u(t))+k_{2}^{\sim}(x, t) N_{2}^{\sim}(v(t))\right) d t
\end{array}\right.
$$

The Adomian polynomials depend only on $u_{0}, v_{0}$; therefor if we can modify the term $\alpha_{1}+\beta_{1} x+$ $L^{-1} f_{1}(x)$ and $\alpha_{2}+\beta_{2} x+L^{-1} f_{2}(x)$ in equation (2), then $u_{0}, v_{0}$ are modified. This can be done by adding a term to both sides of the equation. Let $g_{1}, g_{2}$ be the proper terms, which are determined next; then

$$
\left\{\begin{array}{c}
u(x)+g_{1}=\alpha_{1}+\beta_{1} x+L^{-1} f_{1}(x)+g_{1}+L^{-1} \int_{a}^{b(x)}\left(k_{1}(x, t) N_{1}(u(t))+k_{1}^{\sim}(x, t) N_{1}^{\sim}(v(t))\right) d t \\
v(x)+g_{2}=\alpha_{2}+\beta_{2} x+L^{-1} f_{2}(x)+g_{2}+L^{-1} \int_{a}^{b(x)}\left(k_{2}(x, t) N_{2}(u(t))+k_{2}^{\sim}(x, t) N_{2}^{\sim}(v(t))\right) d t
\end{array}\right.
$$

By applying the modified Adomian method on equation (2), we obtain

$$
\begin{gathered}
\left\{\begin{array}{l}
u_{0}=g_{1} \\
v_{0}=g_{2}
\end{array}\right. \\
\left\{\begin{array}{l}
u_{1}=\alpha_{1}+\beta_{1} x+L^{-1} f_{1}(x)-g_{1}+L^{-1} \int_{a}^{b(x)}\left(k_{1}(x, t) A_{0}(t)+k_{1}^{\sim}(x, t) A_{0}^{\sim}(t)\right) d t \\
v_{1}=\alpha_{2}+\beta_{2} x+L^{-1} f_{2}(x)-g_{2}+L^{-1} \int_{a}^{b(x)}\left(k_{2}(x, t) B_{0}(t)+k_{2}^{\sim}(x, t) B_{0}^{\sim}(v(t))\right) d t
\end{array}\right. \\
\left\{\begin{array}{l}
u_{k+1}=L^{-1} \int_{a}^{b(x)}\left(k_{1}(x, t) A_{k}(t)+k_{1}^{\sim}(x, t) A_{K}^{\sim}(t)\right) d t, \quad k \geq 1 \\
v_{k+1}=L^{-1} \int_{a}^{b(x)}\left(k_{2}(x, t) B_{k}(t)+k_{2}^{\sim}(x, t) B_{K}^{\sim}(v(t))\right) d t, \quad k \geq 1
\end{array}\right.
\end{gathered}
$$


Hence, we present the following algorithm

\subsection{The Algorithm}

Choose small natural numbers $\mathrm{m}, \mathrm{n}$.

Step1: Apply the Adomian method on Eq. (1) and calculate $\left(u_{0}, v_{0}\right),\left(u_{1}, v_{1}\right), \ldots,\left(u_{n}, v_{n}\right)$. Set

$$
\begin{aligned}
& \omega_{1}^{1}=u_{0}+u_{1}+\cdots+u_{n} \\
& \omega_{2}^{1}=v_{0}+v_{1}+\cdots+v_{n}
\end{aligned}
$$

Step2: For $i=2: m$, do

$$
\begin{aligned}
& \omega_{1}^{i-1}=g_{1} \\
& \omega_{2}^{i-1}=g_{2} \\
& \left\{\begin{array}{l}
u_{0}=g_{1} \\
v_{0}=g_{2}
\end{array}\right. \\
& \left\{\begin{array}{l}
u_{1}=\alpha_{1}+\beta_{1} x+L^{-1} f_{1}(x)-g_{1}+L^{-1} \int_{a}^{b(x)}\left(k_{1}(x, t) A_{0}(t)+k_{1}^{\sim}(x, t) A_{0}^{\sim}(t)\right) d t \\
v_{1}=\alpha_{2}+\beta_{2} x+L^{-1} f_{2}(x)-g_{2}+L^{-1} \int_{a}^{b(x)}\left(k_{2}(x, t) B_{0}(t)+k_{2}^{\sim}(x, t) B_{0}^{\sim}(v(t))\right) d t
\end{array}\right. \\
& \left\{\begin{array}{l}
u_{k+1}=L^{-1} \int_{a}^{b(x)}\left(k_{1}(x, t) A_{k}(t)+k_{1}^{\sim}(x, t) A_{K}^{\sim}(t)\right) d t, \quad k \geq 1 \\
v_{k+1}=L^{-1} \int_{a}^{b(x)}\left(k_{2}(x, t) B_{k}(t)+k_{2}^{\sim}(x, t) B_{K}^{\sim}(v(t))\right) d t, \quad k \geq 1
\end{array}\right.
\end{aligned}
$$

Set

$$
\begin{aligned}
& \omega_{1}^{i}=u_{0}+u_{1}+\cdots+u_{n} \\
& \omega_{2}^{i}=v_{0}+v_{1}+\cdots+v_{n}
\end{aligned}
$$

end of for.

Remarks

- $\quad \omega_{1}^{i}, \omega_{2}^{i}$ can be considered as the approximate solution of Eq. (1).

- The Adomian method usually gives the sum of some of the first few terms gives an approximation of $u, v$. In the new algorithm (RADM), we update $u_{0}, v_{0}$ in each step but we do not calculate the terms with large index in each step; therefore, $m$ and $n$ are considered to be small, say, $m=3$ and $n=2$.

- Applied the restarted Adomian method in $m$ steps; and in each step, we obtained $n$ terms of standard Adomian method with updated $u_{0}, v_{0}$. Note that we used $A_{0}, A_{1}$. . . , $A_{n-1}, A_{0}^{\sim}, A_{1}^{\sim}, \ldots, A_{n-1}^{\sim}, B_{0}, B_{1} \ldots, B_{n-1}, B_{0}^{\sim}, B_{1}^{\sim}, \ldots, B_{n-1}^{\sim}$ in each step, whereas for the standard Adomian method, we obtained $m n$ terms, i.e., used $A_{0}, A_{1}, \ldots$, $A_{m n-1}, A_{0}^{\sim}, A_{1}^{\sim}, \ldots, A_{m n-1}^{\sim}, B_{0}, B_{1}, \ldots, B_{m n-1}, B_{0}^{\sim}, B_{1}^{\sim}, \ldots, B_{m n-1}^{\sim}$.

\section{Computational Results and Analysis}

\section{Example 1}

Consider the system of nonlinear Volterra integro differential equation [8]

$$
\begin{cases}u^{\prime}(x)=1-x+\frac{x^{2}}{2}-\frac{x^{4}}{12}+\int_{0}^{x}\left((x-t) u^{2}+v^{2}\right) d t, & u(0)=1 \\ v^{\prime}(x)=-1-x-\frac{3 x^{2}}{2}-\frac{x^{4}}{12}+\int_{0}^{x}\left(u^{2}+(x-t) v^{2}\right) d t, & v(0)=1\end{cases}
$$


With the exact solution $(1+x, 1-x)$. Applying $L^{-1}()=.\int_{0}^{x}() d$.$x to the both sides gives,$

$$
\left\{\begin{array}{l}
u(x)=1+x-\frac{x^{2}}{2}+\frac{1}{6} x^{3}-\frac{x^{5}}{60}+L^{-1} \int_{0}^{x}\left((x-t) u^{2}+v^{2}\right) d t \\
v(x)=1-x-\frac{x^{2}}{2}-\frac{x^{3}}{2}-\frac{x^{5}}{60}+L^{-1} \int_{0}^{x}\left(u^{2}+(x-t) v^{2}\right) d t
\end{array}\right.
$$

By applying the new algorithm with $n=2$ and $m=2$, we obtain:

Step 1

$$
\begin{aligned}
& \left\{\begin{array}{l}
u_{0}=1+x-\frac{x^{2}}{2}+\frac{1}{6} x^{3}-\frac{x^{5}}{60} \\
v_{0}=1-x-\frac{x^{2}}{2}-\frac{x^{3}}{2}-\frac{x^{5}}{60} \\
u_{k+1}=L^{-1} \int_{0}^{x}\left((x-t) A_{k}(t)+B_{k}(t)\right) d t, \quad k \geq 0 \\
v_{k+1}=L^{-1} \int_{0}^{x}\left(A_{k}(t)+(x-t) B_{k}(t)\right) d t, \quad k \geq 0
\end{array}\right. \\
& \left\{\begin{array}{l}
u_{1}=\frac{1}{2} x^{2}-\frac{1}{6} x^{3}+\frac{1}{12} x^{4}+\frac{13}{360} x^{6}+\cdots \\
v_{1}=\frac{1}{2} x^{2}+\frac{1}{2} x^{3}-\frac{1}{12} x^{4}-\frac{1}{30} x^{5}+\cdots \\
u_{2}=\frac{1}{12} x^{4}+\frac{1}{60} x^{5}-\frac{1}{20} x^{6}-\frac{31}{1260} x^{7}+\cdots \\
v_{2}=\frac{1}{12} x^{4}+\frac{1}{20} x^{5}-\frac{1}{45} x^{6}+\frac{1}{252} x^{7}+\cdots \\
\vdots
\end{array}\right. \\
& \omega_{1}{ }^{1}=u_{0}+u_{1}+u_{2}=1+x+\frac{1}{6} x^{4}-\frac{1}{72} x^{6}-\frac{3}{280} x^{7}+\cdots
\end{aligned}
$$

Step 2

$$
\begin{aligned}
& \left\{\begin{array}{c}
u_{0}=1+x+\frac{1}{6} x^{4}-\frac{1}{72} x^{6}-\frac{3}{280} x^{7}+\cdots \\
v_{0}=1-x-\frac{1}{360} x^{6}+\frac{13}{2520} x^{7}+\cdots \\
u_{1}=1+x-\frac{x^{2}}{2}+\frac{1}{6} x^{3}-\frac{x^{5}}{60}-\left(1+x+\frac{1}{6} x^{4}-\frac{1}{72} x^{6}-\frac{3}{280} x^{7}+\cdots\right) \\
\quad+L^{-1} \int_{0}^{x}\left((x-t) A_{0}(t)+B_{0}(t)\right) d t
\end{array}\right. \\
& \begin{array}{c}
v_{1}=1-x-\frac{x^{2}}{2}-\frac{x^{3}}{2}-\frac{x^{5}}{60}-\left(1-x-\frac{1}{360} x^{6}+\frac{13}{2520} x^{7}+\cdots\right)+ \\
\quad+L^{-1} \int_{0}^{x}\left(A_{0}(t)+(x-t) B_{0}(t)\right) d t
\end{array} \\
& \begin{array}{c}
u_{k+1}=L^{-1} \int_{0}^{x}\left((x-t) A_{k}(t)+B_{k}(t)\right) d t, \quad k \geq 1 \\
v_{k+1}=L^{-1} \int_{0}^{x}\left(A_{k}(t)+(x-t) B_{k}(t)\right) d t, \quad k \geq 1
\end{array} \\
& \left\{\begin{array}{c}
u_{1}=\frac{1}{72} x^{6}+\frac{31}{2520} x^{7}+\frac{1}{2016} x^{8}-\frac{73}{90720} x^{9}+\cdots \\
v_{1}=\frac{1}{72} x^{6}+\frac{1}{360} x^{7}+\frac{1}{480} x^{8}-\frac{73}{30240} x^{9}+\ldots \\
u_{2}=\frac{1}{2016} x^{8}-\frac{23}{90720} x^{9}+\frac{13}{226800} x^{10}-\frac{31}{554400} x^{11}+\cdots \\
v_{2}=\frac{1}{2016} x^{8}+\frac{71}{90720} x^{9}+\frac{23}{90720} x^{10}-\frac{1}{142560} x^{11}+\cdots \\
\quad
\end{array}\right. \\
& \begin{array}{c}
\omega_{1}^{2}=u_{0}+u_{1}+u_{2}=1+x+\frac{1}{6} x^{4}+\frac{1}{630} x^{7}+\frac{1}{720} x^{8}+\cdots \\
\end{array}
\end{aligned}
$$




$$
\omega_{2}^{2}=v_{0}+v_{1}+v_{2}=1-x+\frac{1}{90} x^{6}+\frac{1}{126} x^{7}+\cdots
$$

The results produced by the present method with only few components $(m=2)$ are in a very good agreement with the best of the results of the methods listed in Table (1-a) and Table (1-b). The restarted Adomian method solution compared with exact solution in Fig. (1-a) and Fig. (1-b).

Table (1-a). Comparison between Exact Solution $u(x)$ and Approximate Solution Using Method (RADM)

\begin{tabular}{|c|c|c|c|}
\hline$X$ & Exact & (RADM) & Absolute Error \\
\hline 0.00 & 1.00000000 & 1.00000000 & $0.000000000 \mathrm{e}+00$ \\
\hline 0.10 & 1.10000000 & 1.10001667 & $1.66670000 \mathrm{e}-05$ \\
\hline 0.20 & 1.20000000 & 1.20026669 & $2.66691000 \mathrm{e}-04$ \\
\hline 0.30 & 1.30000000 & 1.30135044 & $1.35043500 \mathrm{e}-03$ \\
\hline 0.40 & 1.40000000 & 1.40427014 & $4.27013700 \mathrm{e}-03$ \\
\hline 0.50 & 1.50000000 & 1.51043416 & $1.04341590 \mathrm{e}-02$ \\
\hline
\end{tabular}

Table (1-b). Comparison between Exact Solution $v(x)$ and Approximate Solution Using Method (RADM)

\begin{tabular}{|c|c|c|c|}
\hline$X$ & Exact & M5 & Absolute Error \\
\hline 0.00 & 1.00000000 & 1.00000000 & $0.00000000 \mathrm{e}+00$ \\
\hline 0.10 & 0.90000000 & 0.90000001 & $1.19000000 \mathrm{e}-08$ \\
\hline 0.20 & 0.80000000 & 0.80000081 & $8.12700000 \mathrm{e}-07$ \\
\hline 0.30 & 0.70000000 & 0.70000984 & $9.83950000 \mathrm{e}-06$ \\
\hline 0.40 & 0.60000000 & 0.60005857 & $5.85747000 \mathrm{e}-05$ \\
\hline 0.50 & 0.50000000 & 0.50023614 & $2.36135000 \mathrm{e}-04$ \\
\hline
\end{tabular}

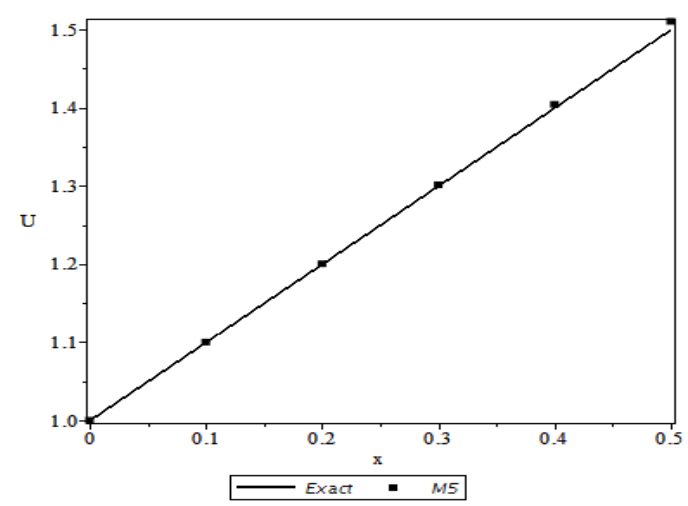

Fig. (1-a). Comparison between exact solution $u(x)$ and approximate solution using method (RADM).

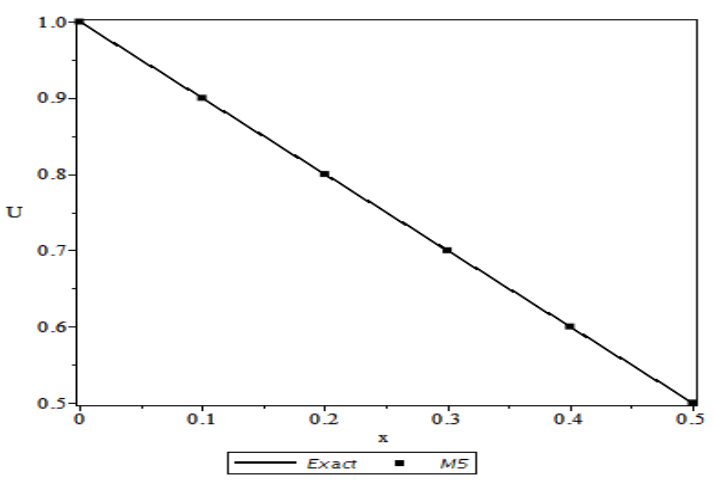

Fig. (1-b). Comparison between exact solution $v(x)$ and approximate solution using method (RADM).

\section{Example 2}


Consider the system of nonlinear Fredholm integro differential equation

$$
\begin{cases}u^{\prime \prime}(x)=2+\frac{12}{5} x-\int_{0}^{1} x\left(u^{2}+v^{2}\right) d t, & u(0)=1, u^{\prime}(0)=0 \\ v^{\prime \prime}(x)=-2+\frac{4}{3} x-\int_{0}^{1} x\left(u^{2}-v^{2}\right) d t, & u(0)=1, u^{\prime}(0)=0\end{cases}
$$

With the exact solution $\left(1+x^{2}, 1-x^{2}\right)$.

Applying $L^{-1}()=.\int_{0}^{x} \int_{0}^{x}() d x d$.$x to the both sides gives,$

$$
\left\{\begin{array}{l}
u(x)=1+x^{2}+\frac{12}{30} x^{3}-L^{-1} \int_{0}^{1} x\left(u^{2}+v^{2}\right) d t \\
v(x)=1-x^{2}+\frac{4}{18} x^{3}-L^{-1} \int_{0}^{1} x\left(u^{2}-v^{2}\right) d t
\end{array}\right.
$$

By applying the new algorithm with $n=2$ and $m=2$, we obtain:

Step 1

Step2

$$
\begin{aligned}
& \begin{cases}u_{0}=1+x^{2}+\frac{12}{30} x^{3} & \\
v_{0}=1-x^{2}+\frac{4}{1 x} x^{3} & \\
u_{k+1}=-L^{-1} \int_{0}^{1} x\left(A_{k}+B_{k}\right) d t, & k \geq 0 \\
v_{k+1}=-L^{-1} \int_{0}^{1} x\left(A_{k}-B_{k}\right) d t, & k \geq 0\end{cases} \\
& \left\{\begin{array}{c}
u_{1}=-\frac{19847}{42525} x^{3} \\
v_{1}=-\frac{1666}{6075} x^{3} \\
u_{2}=\frac{13539649}{160744500} x^{3} \\
v_{2}=\frac{10157669}{160744500} x^{3} \\
\vdots
\end{array}\right. \\
& \omega_{1}{ }^{1}=u_{0}+u_{1}+u_{2}=1+x^{2}+\frac{2815789}{160744500} x^{3} \\
& \omega_{2}{ }^{1}=v_{0}+v_{1}+v_{2}=1-x^{2}+\frac{1796309}{160744500} x^{3}
\end{aligned}
$$

$$
\left\{\begin{array}{c}
u_{0}=1+x^{2}+\frac{2815789}{160744500} x^{3} \\
v_{0}=1-x^{2}+\frac{1796309}{160744500} x^{3} \\
u_{1}=1+x^{2}+\frac{12}{30} x^{3}-\left(1+x^{2}+\frac{2815789}{160744500} x^{3}\right) \\
\quad-L^{-1} \int_{0}^{1} x\left(A_{0}(t)+B_{0}(t)\right) d t \\
v_{1}=1-x^{2}+\frac{4}{18} x^{3}-\left(1-x^{2}+\frac{1796309}{160744500} x^{3}\right)+ \\
\quad-L^{-1} \int_{0}^{1} x\left(A_{0}(t)-B_{0}(t)\right) d t \\
u_{k+1}=-L^{-1} \int_{0}^{1} x\left(A_{k}+B_{k}\right) d t, \quad k \geq 1 \\
v_{k+1}=-L^{-1} \int_{0}^{1} x\left(A_{k}-B_{k}\right) d t, \quad k \geq 1
\end{array}\right.
$$$$
\left\{\begin{array}{r}
u_{1}=-0.02027080626 x^{3} \\
v_{1}=-0.01330179127 x^{3} \\
u_{2}=0.003208871286 x^{3} \\
v_{2}=0.002455726061 x^{3} \\
:
\end{array}\right.
$$ 


$$
\begin{aligned}
& \omega_{1}^{2}=u_{0}+u_{1}+u_{2}=1+x^{2}+0.0004552366791 x^{3} \\
& \omega_{2}{ }^{2}=v_{0}+v_{1}+v_{2}=1-x^{2}+0.0003288676839 x^{3}
\end{aligned}
$$

The results produced by the present method with only few components $(m=2)$ are in a very good agreement with the best of the results of the methods listed in Table (2-a) and Table (2-b). The (RADM) solution compared with exact solution in Fig. (2-a) and Fig. (2-b).

Table (2-a). Comparison between Exact Solution $u(x)$ and Approximate Solution Using Method (RADM)

\begin{tabular}{|c|c|c|c|}
\hline$X$ & Exact & M5 & Absolute Error \\
\hline 0.00 & 1.00000000 & 1.00000000 & $0.00000000 \mathrm{e}+00$ \\
\hline 0.10 & 1.01000000 & 1.01000046 & $4.55000000 \mathrm{e}-07$ \\
\hline 0.20 & 1.04000000 & 1.04000364 & $3.64200000 \mathrm{e}-06$ \\
\hline 0.30 & 1.09000000 & 1.09001229 & $1.22910000 \mathrm{e}-05$ \\
\hline 0.40 & 1.16000000 & 1.16002914 & $2.91350000 \mathrm{e}-05$ \\
\hline 0.50 & 1.25000000 & 1.25005690 & $5.69050000 \mathrm{e}-05$ \\
\hline
\end{tabular}

Table (2-b). Comparison between exact solution $v(x)$ and Approximate Solution Using Method (RADM)

\begin{tabular}{|c|c|c|c|}
\hline$X$ & Exact & M5 & Absolute Error \\
\hline 0.00 & 1.00000000 & 1.00000000 & $0.00000000 \mathrm{e}+00$ \\
\hline 0.10 & 0.99000000 & 0.99000033 & $3.28900000 \mathrm{e}-07$ \\
\hline 0.20 & 0.96000000 & 0.96000263 & $2.63090000 \mathrm{e}-06$ \\
\hline 0.30 & 0.91000000 & 0.91000888 & $8.87940000 \mathrm{e}-06$ \\
\hline 0.40 & 0.84000000 & 0.84002105 & $2.10475000 \mathrm{e}-05$ \\
\hline 0.50 & 0.75000000 & 0.75004111 & $4.11085000 \mathrm{e}-05$ \\
\hline
\end{tabular}

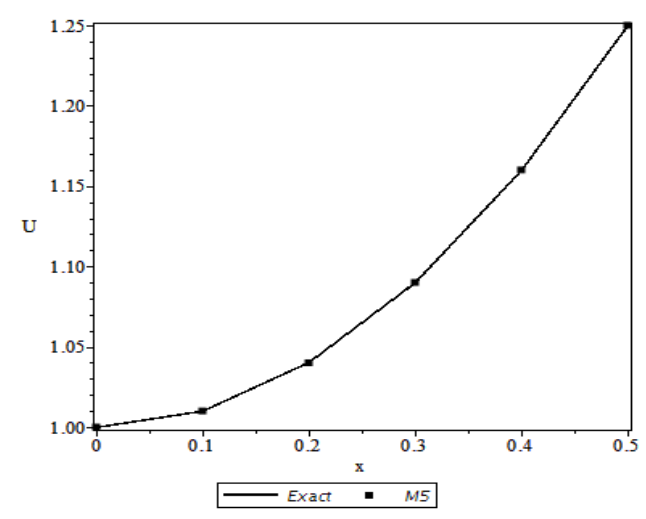

Fig. (2-a). Comparison between exact solution $u(x)$ and approximate solution using method (RADM).

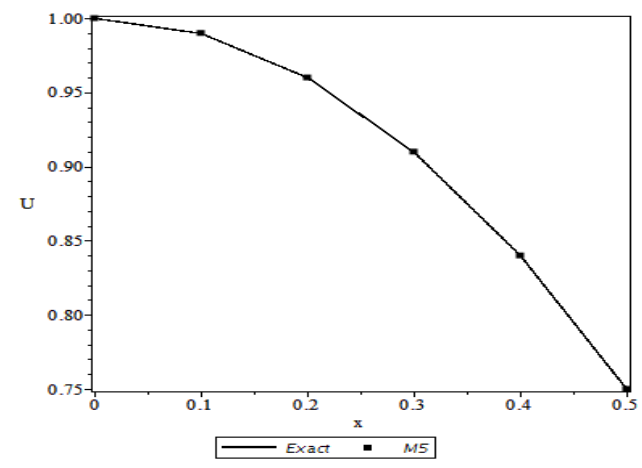

Fig. (2-b). Comparison between exact solution $v(x)$ and approximate solution using method (RADM). 


\section{Conclusion}

The present modification of Adomian decomposition method (RADM) has been successfully applied in this paper to finding the approximate solutions of system of nonlinear integro differential equations. The investigated examples show that better accuracy was achieved via the present method.

\section{References}

[1] Adomian, G. (1994). Solving Frontier Problems of Physics: The Decomposition Method. Kluwer Academic Publisher, Boston, MA.

[2] Adomian, G. (1996). Modified adomian polynomials. Mathe-matical Computation, 76(1), 95-97.

[3] Babolian, E., et al. (2003). Restarted adomian method for algebraic equations. Appl. Math. Comput., 146, 533-541.

[4] Alireza, V., et al. (2008). Restarted adomian method for solving system of nonlinear equations. Journal of Applied Mathematics, 5(17).

[5] Babolian, E., et al. (January 2005). Restarted adomian method for nonlinear differential equations. International Journal of Computer Mathematics, 82(1), 97-102.

[6] Babolian, E., et al. (2004). Restarted adomian method for integral equations. Applied Mathematics and Computation, 153, 353-359.

[7] Babolian, E., et al. (2005). Restarted adomian method for system of nonlinear voltra integral equations. APP. Math Comput., 161, 745-751.

[8] Wazwaz. (2011). Linear and Nonlinear Integral Equations Methods and Applications. Beijing and Springer.

H. O. Bakodah is a professor of mathematics at Faculty of Science-AL Faisaliah Campus, King Abdulaziz University, Saudi Arabia. She received the PhD degree in "numerical analysis" at College of education for Girls Scientific Section Jeddah, Saudi Arabia. Her main research interests are: numerical methods for solving differential equations and integral equations.

M. Al-Mazmumy is assistant professor of mathematics at Faculty of Science-AL Faisaliah Campus, King Abdulaziz University, Saudi Arabia. She received the PhD degree in "differential equations" at College of education for Girls Scientific Section Jeddah, Saudi Arabia. Her main research interests are ordinary differential equations, partial differential equations, integral equations, stability and oscillation of predatorprey models and adomian decomposition method.

S. O. Almuhalbedi is master student in mathematics at King Abdulaziz University, Saudi Arabia. She received the bachelor degree in mathematics at Faculty of Science for Girls, King Abdulaziz University, Jeddah, Saudi Arabia. Her main research interests are: differential equations, integral equations and applied mathematics. 